AFRL-IF-RS-TR-1998-205

Final Technical Report

November 1998

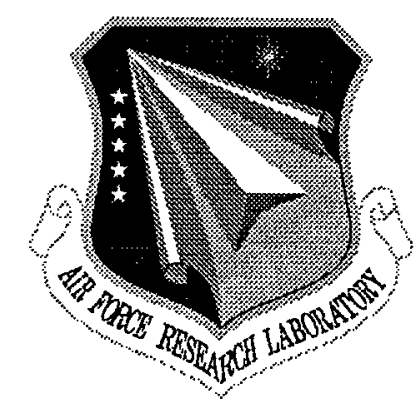

\title{
HAND-HELD COMPUTING DEVICES AND LARGE KNOWLEDGE BASES
}

Capraro Technologies, Inc.

Gerard T. Capraro

APPROVED FOR PUBLIC RELEASE; DISTRIBUTION UNLIMITED.
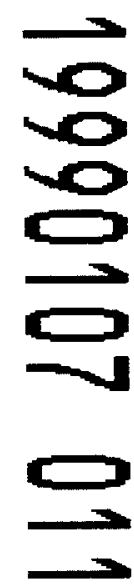

\section{AIR FORCE RESEARCH LABORATORY INFORMATION DIRECTORATE ROME RESEARCH SITE ROME, NEW YORK}


This report has been reviewed by the Air Force Research Laboratory, Information Directorate, Public Affairs Office (IFOIPA) and is releasable to the National Technical Information Service (NTIS). At NTIS it will be releasable to the general public, including foreign nations.

AFRL-IF-RS-TR-1998-205 has been reviewed and is approved for publication.

APPROVED:

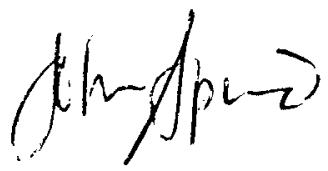

JOHN SPINA

Project Engineer

FOR THE DIRECTOR:

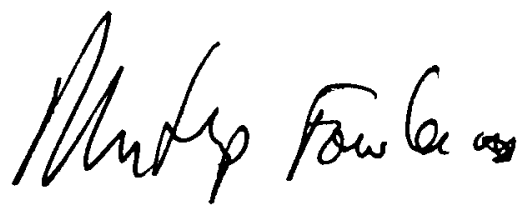

NORTHRUP FOWLER, III, Technical Advisor Information Technology Division Information Directorate

If your address has changed or if you wish to be removed from the Air Force Research Laboratory Rome Research Site mailing list, or if the addressee is no longer employed by your organization, please notify AFRL/IFTB, 525 Brooks Rd., Rome, NY 13441-4505. This will assist us in maintaining a current mailing list.

Do not return copies of this report unless contractual obligations or notices on a specific document require that it be returned. 


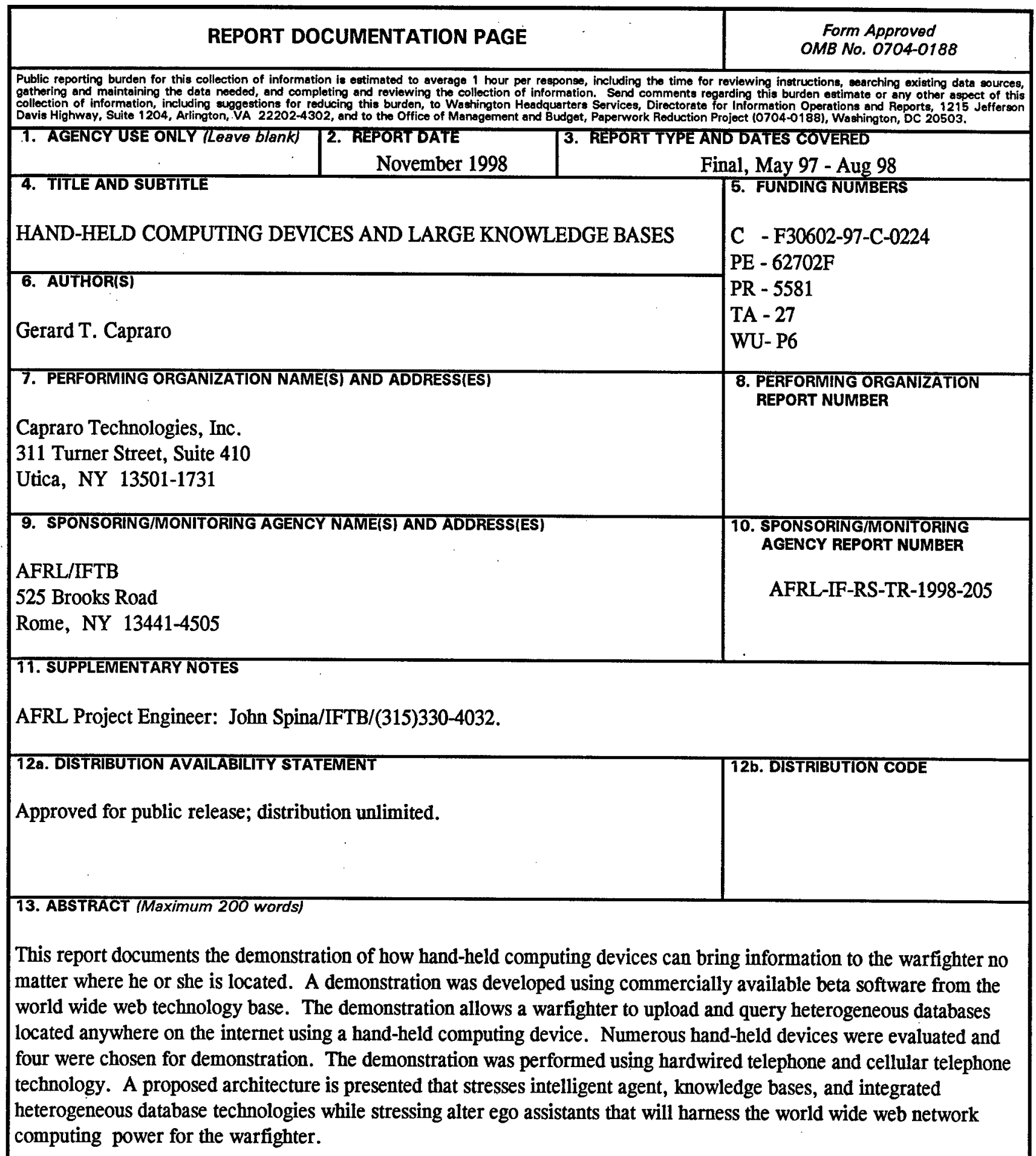




\section{TABLE OF CONTENTS}

\section{Page}

Abstract 1

1. Introduction 1

2. Effort Description 2

3. Hand-Held Computing Devices 4

4. Demonstration 5

5. Preliminary Findings and New Network Technology 9 


\section{List of Figures}

\section{Page}

Figure 1. Demonstration

3

Figure 2. HCD Evaluation Attributes 4

Figure 3. Hand-Held Computing Devices 5

Figure 4. HCD Main Menu $\quad 7$

Figure 5. Database Query 8

Figure 6. Database Query Result 8

Figure 7. Database Update 9

Figure 8. Preliminary Architecture 11 


\begin{abstract}
This report documents the demonstration of how hand-held computing devices can bring information to the war fighter no matter where he or she is located. A demonstration is developed using commercially available beta software from the world wide web technology base. The demonstration allows a war fighter to upload and query heterogeneous databases located anywhere on the Internet using a hand-held computing device. Numerous hand-held devices were evaluated and four were chosen for demonstration. The demonstration was performed using hardwired telephone and cellular telephone technology. A proposed architecture is presented that stresses intelligent agent, knowledge bases, and integrated heterogeneous database technologies while stressing alter ego assistants that will harness the world wide web network computing power for the war fighter.
\end{abstract}

\title{
1.0 Introduction
}

Accurate and timely data is a corporate resource. Inaccurate or untimely data is a corporate expense. The military lacks the ability to provide timely, accurate data and information to its war fighters because the war fighter does not have access to computer technology in the combat environment. The Department of Defense is data rich and information poor. War fighters in many situations must labor over an inordinate amount of data before obtaining data or information they require.

Rome Laboratory and now the USAF Research Laboratory's Information Directorate has been investigating solutions to these problems, through the integration of large databases and knowledge bases. They have pursued numerous efforts by many experienced researchers. It is now time to provide access to this wealth of data and information from anywhere in the world.

Military personnel need to communicate with superiors and command centers. Over the years these communications have been primarily audio. However, with advances in computing and communications technologies, it is possible to exchange both audio and visual communications with anyone using laptop computers, personal digital assistants, and consumer electronics devices. One can stay in touch with his/her e-mail, send or receive faxes, access applications on a home computer, and query knowledge and databases anywhere in the world. He/she can have access to very large amounts of data and information in any form (i.e. voice, graphics, and video).

The objective of this effort was to perform a proof of feasibility demonstration by remotely accessing disparate databases in a seamless manner via the Internet using a hand-held computing device (HCD). A demonstration was performed by using both a cellular phone and telephone connection to the world wide web (www). This demonstration provided not only the proof that this concept is feasible but it has also uncovered gaps in the technology that need attention before a realistic system can be fielded.

Universal goals are:

- To provide field acquisition and retrieval of quality data in a timely manner.

- To upload, download, and store multimedia data, i.e. pictures, sound, and video. 
- To search distributed databases and knowledge bases in "real time" and in a secure mode.

- To upload, download, store, fuse and present sensor data in a meaningful and intuitive manner.

This effort provides a demonstration of a necessary portion of the foundation required to meet these goals.

The next section, Section 2, provides a description of and motivation for this effort. Section 3 discusses the HCDs purchased at the beginning and end of the effort. Section 4 describes the demonstration performed. Section 5 documents preliminary findings and a view of the next generation of network technology to support HCDs and their processing needs.

\subsection{Effort Description}

Legacy databases exist throughout the US Air Force. These databases were costly to develop and are costly to maintain. They are the backbone to many military decision processes utilized throughout the US Air Force, during both peace and conflict. The Air Force has pursued research to integrate these databases in such a manner that these databases appear to the user to be on the same machine, and using the same database management system (DBMS). This capability allows the individual databases to continue to be developed and maintained in their current consistent manner and yet allow many users to integrate their data and reap the benefits of this synergy.

The development of the browser technology, the Internet, and Intranet systems provide the capability of integrating these disparate databases with the same user interface. This capability is independent and transparent of the DBMS and, to a certain extent, its resident machine. This allows a user to interact with these integrated databases over a network as if they were a homogeneous database.

This effort investigated a vendor neutral approach. An integrated approach was performed, using Java and Hypertext Mark Up Language (HTML) as the languages for building the user front-end and Java's database connectivity (JDBC) for accessing different DBMSs was used. Java programs can run on a Java virtual machine within a browser or as a native installation.

The motivation for this effort and the use of HCDs was to bring the capability of this technology to the war fighter. There is no reason why the power of a network of computers cannot ultimately be brought to the battlefield. As the telephone can be used by anyone speaking any language, a HCD can provide anyone access to data and information. In this manner, an HCD can be used by a physician or medic that scans an injured war fighter's dog tag to gain access to the person's medical records, $\mathrm{X}$-rays, MRIs, etc. They can also upload data related to current injuries sustained by the fighter. This same HCD can be used by the commander of the unit to obtain data related to mission planning, staffing, and reconnaissance.

There are many issues that need to be answered before today's HCDs are deployed to every war fighter. This effort's hope was to show the capability of the Internet to handle one aspect of the above scenario: the ability to access two different databases located on two different computer nodes on the Internet. The demonstration should allow the 
user to upload data and retrieve data from the databases such that the DBMS "appears" resident on the HCD. The user interface to the database must be simple and intuitive. The object was to make it easy to understand, easy to use, and as keyboard independent as possible.

The advent of the modern day computer, the Internet, and arcade games have conditioned today's war fighter to be at ease with computer technology. However, war fighters are not computer programmers and there are no database administrators in the field to help them formulate a new query that wasn't pre-programmed. The approach taken was based on a simple database application for demonstration purposes. By keeping the database application simple, evaluating the underlying technology was not clouded by the complexity of the application chosen.

The experiment we chose is illustrated in Figure 1. Once logged into the network via a phone line or cellular connection the user is able to either retrieve data from or submit data to one or both databases (A or B) located on two different nodes on the Internet. The user sees the integrated view of the data. The host node through which the user accesses the Internet recognizes the user and sends HTML pages. The user can then navigate around the page, enter parameters, submit queries and view query results. These queries can be used to view, update, append or delete records. All of the data processing is done on the nodes within the network. The HCD is providing the connection to the network, the protocols for communication, the display of the HTML pages, and the capturing of the data and controls from the user.

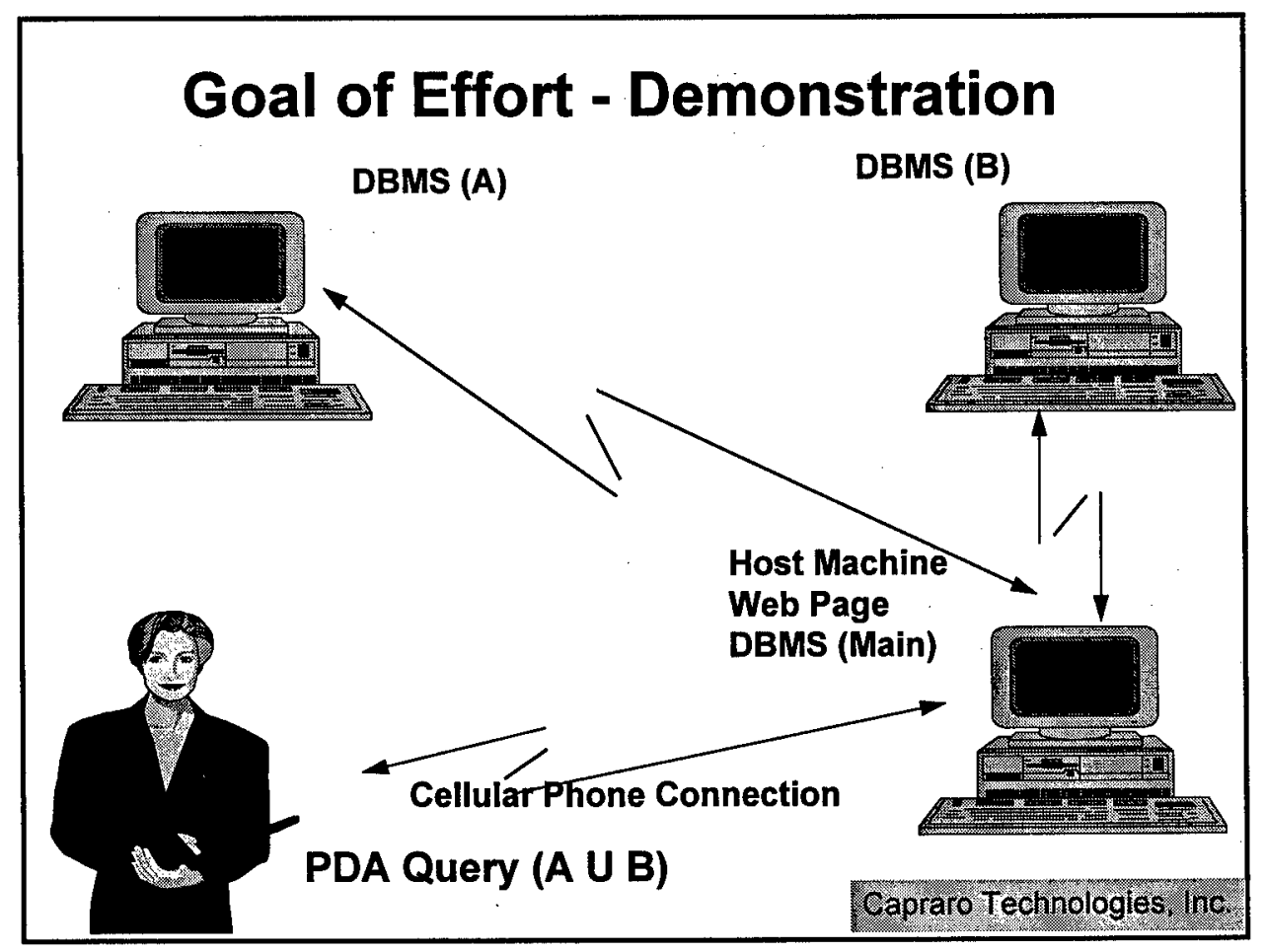

Figure 1. Demonstration Depiction 
The host machine contains the software to communicate with the HCD and the two nodes contain DBMS A and B. It also contains a DBMS with a meta database of the data dictionaries/directories of the databases located within DBMS $A$ and $B$ and the integrated view of these two databases. When the user wishes to query the integrated database, the software running on the host machine recognizes the query and spawns one or more queries to A and/or B, obtains their results, joins them if needed, and performs a projection and translation to comply with the user's integrated view. A similar process is performed if the user changes the contents of the database or adds new occurrances to his/her database.

\subsection{Hand-Held Computing Devices}

When this effort began, CTI purchased four HCDs, two of one kind and two of another. CTI has been tracking HCDs ever since the first Apple Newton MessagePad was available and are aware of the dynamics in the industry. To leverage resources $\mathrm{CTI}$ purchased two HCDs at the beginning of the effort and re-evaluated the technology and purchased the second two at the end of the effort. CTI evaluated the best of them based upon the attributes shown in Figure 2.

\begin{tabular}{|l|l|l|}
\hline Processor speed, & Operating system, & Amount of RAM, \\
\hline Amount of storage, & Expandability, & Overall size, \\
\hline Screen size and type, & Methods for entering data, & $\begin{array}{l}\text { Keyboard type and } \\
\text { availability, }\end{array}$ \\
\hline Battery power, & Modem speeds, & Weight, \\
\hline Hardness or ruggedization, & $\begin{array}{l}\text { Connectivity to other } \\
\text { machines, }\end{array}$ & Communications, \\
\hline Software, and & Price. & \\
\hline
\end{tabular}

Figure 2. HCD Evaluation Attributes

Given the results of this evaluation and the contents of technical articles, Apple's Newton Message Pad 2000 and Philips' Velo 1 were chosen. At the end of the effort CTI re-evaluated the technology and purchased Philips' Velo 500 and Hitachi's HPW $200 E C$. A list of the set of devices evaluated are presented in Figure 3. (Note the data in Figure 3 were obtained in June 1998.) The technology has progressed; processing power has increased, color is now available, memory has increased, better operating systems are available, and HCD prices are coming down and their value is going up. Some HCDs connect to the web, have browser capability, have voice input capability, and they will soon be able to execute Java outside of a browser. 


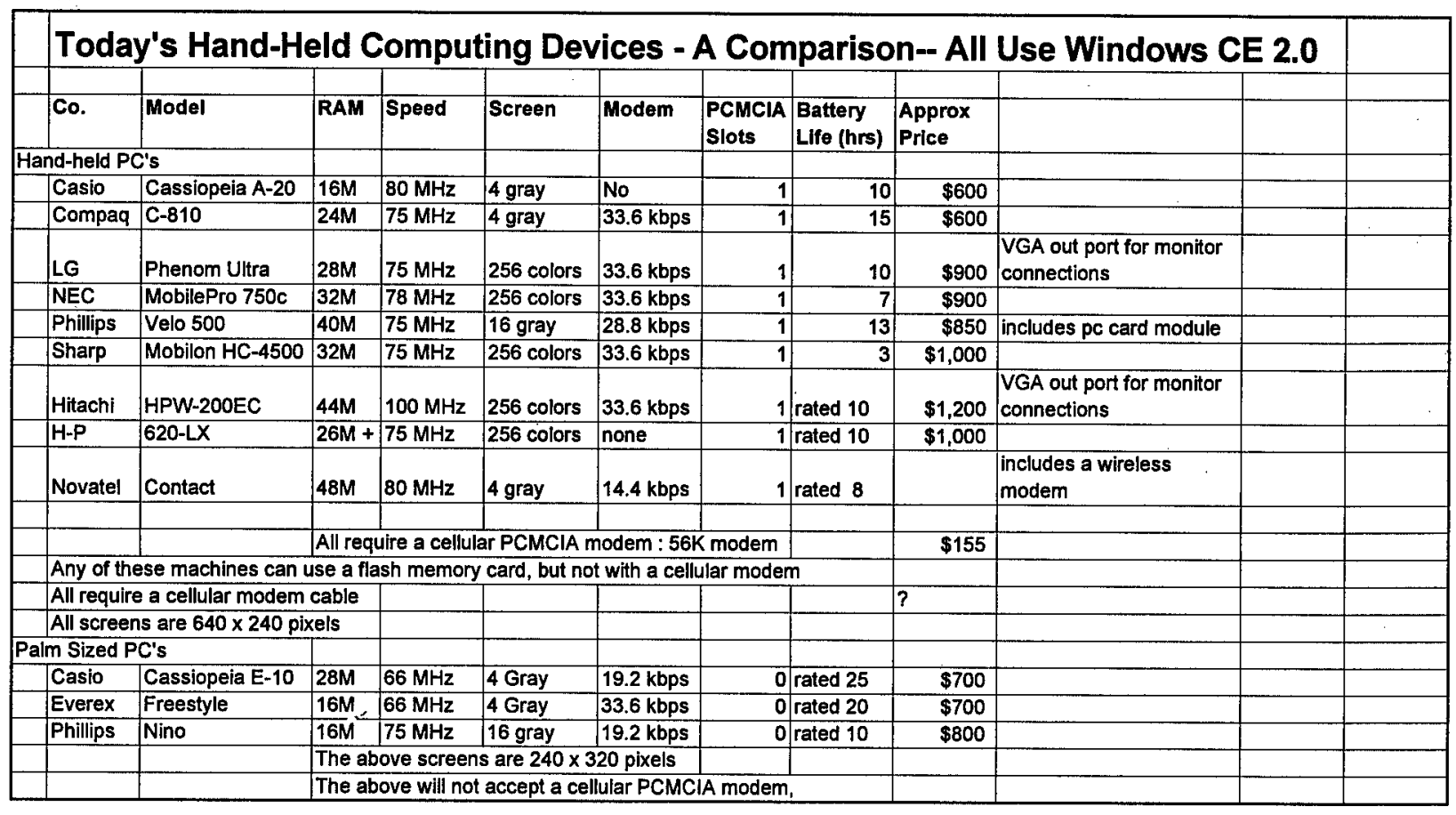

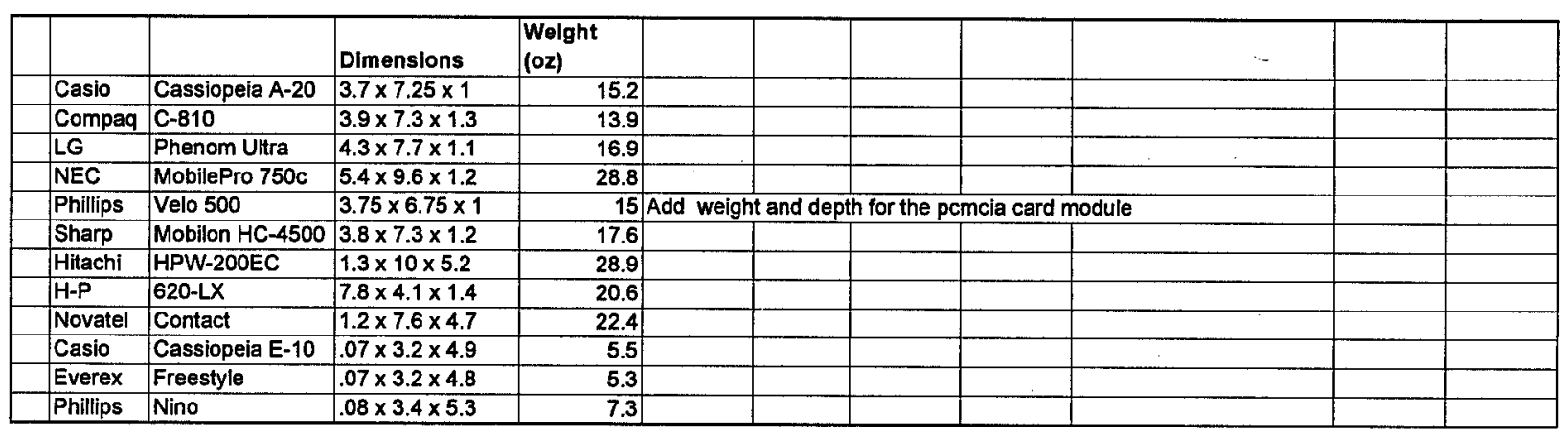

Figure 3. Hand-Held Computing Devices

\subsection{Demonstration}

The demonstration chosen uses very simple database schemas. However, the goal of the demonstration was to show the technology's potential. Two databases were defined, each containing one relation. They are both defined and resident on two different relational DBMSs on two different computers accessible via the world wide web. The HCD calls a node on the internet, i.e. an internet service provider (ISP), which then provides a connection to the computer containing a relational DBMS that contains a meta database defining the data dictionary of the two relations stored on the other two machines. This machine contains the Java code that generates the HTML pages for the HCD and communicates with the user to generate the queries to the other two DBMSs. 
Each database contains a single relation. The schema for $A$ is the Employee Address relation, and For B is the Employee Department relation. These relations are defined below.

\begin{tabular}{|l|}
\hline EmployeeAddress \\
\hline EmployeelD \\
\hline FirstName \\
\hline LastName \\
\hline SpouseFirstName \\
\hline StreetAddress \\
\hline City \\
\hline StateOrProvince \\
\hline PostalCode \\
\hline HomePhone \\
\hline
\end{tabular}

\begin{tabular}{|l|}
\hline EmployeeDepartment \\
\hline EmployeelD \\
\hline SocialSecurityNumber \\
\hline DepartmentNumber \\
\hline
\end{tabular}

Here are sample data for each database:

Sample Employee Address relations.

\begin{tabular}{|l|l|l|l|l|}
\hline EmployeelD & FirstName & LastName & SpouseFirstName & StreetAddress \\
\hline 150 & John & Jones & Rose & State St. \\
\hline 160 & Phil & Jones & Lisa & Robert Rd. \\
\hline
\end{tabular}

\begin{tabular}{|l|l|l|l|}
\hline City & StateOrProvince & PostalCode & HomePhone \\
\hline Rome & NY & 13440 & $315336-4968$ \\
\hline Rome & NY & 13440 & $315336-4987$ \\
\hline
\end{tabular}

Sample Employee Department relations.

\begin{tabular}{|l|l|l|}
\hline EmployeeID & SocialsecurityNumber & DepartmentNumber \\
\hline 150 & 786543332 & 01 \\
\hline 160 & 537675555 & 05 \\
\hline 090 & 352136478 & 05 \\
\hline
\end{tabular}

To link data between the two relations, a join is performed between the two relations on their common primary key, EmployeelD. The Employee Department relation and its occurrences comprise Database $A$ and Employee Department relation and its occurrences comprise Database $B$. The schemas of both databases and their internet addresses are stored on the Host Machine as shown in Figure 1.

Our demonstration consists of a select query on the joining of the two databases related to an employee's last name and their resident city and a method for the user to enter a subset of the attributes for a new employee entering the company. A screen dump of the first screen is shown in Figure 4. 


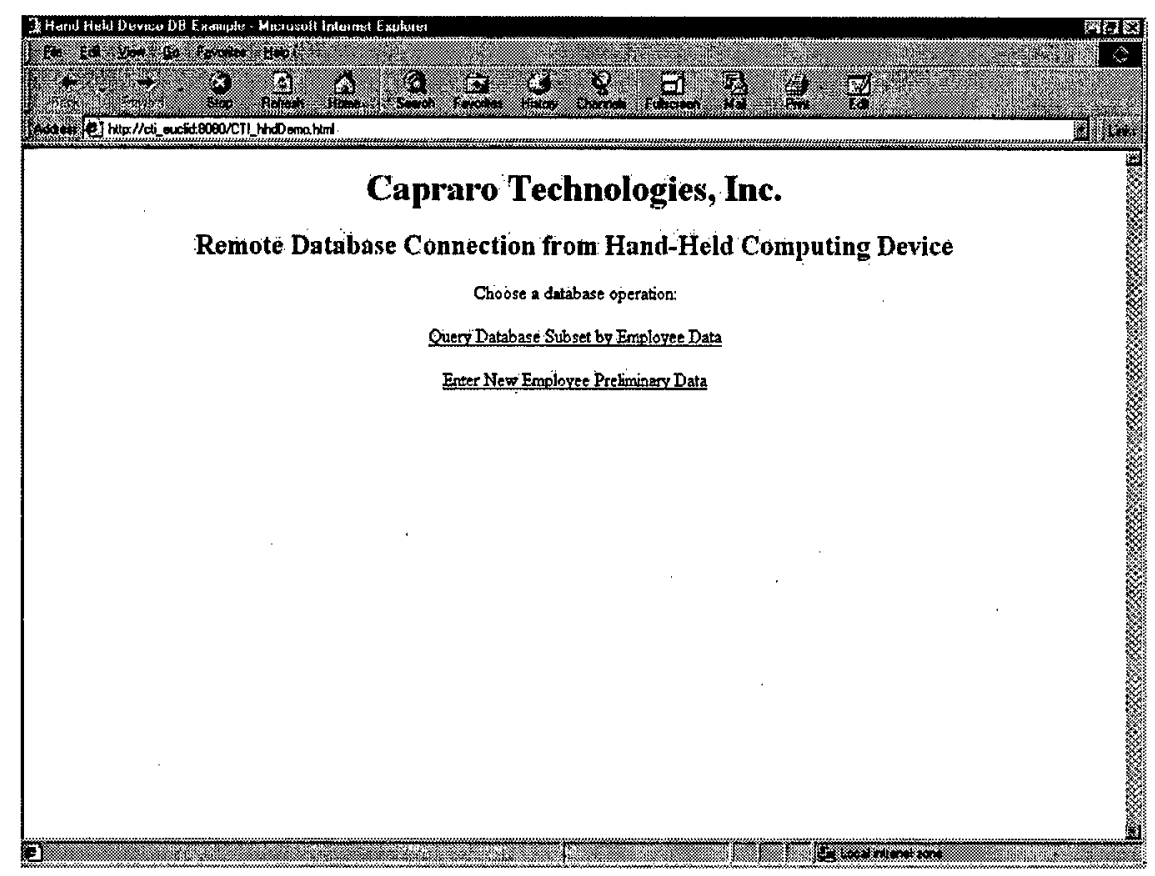

Figure 4. HCD Main Menu

The screens shown in Figures 5 and 6 allow the user to create a query to the databases. From the first pull-down box the user selects either LastName, City, SocialSecurityNumber or EmployeeID from the list. The user then chooses a comparison from the second pull-down box, either equality $(=)$, or if the field selected is numeric, greater than, $(>)$ or less than $(<)$ may be selected. The next box accepts the value for this comparison. The user can then add additional lines to the query, and connect these lines by a logical AND or OR. A SQL query statement is generated from these lines and submitted to the database. 


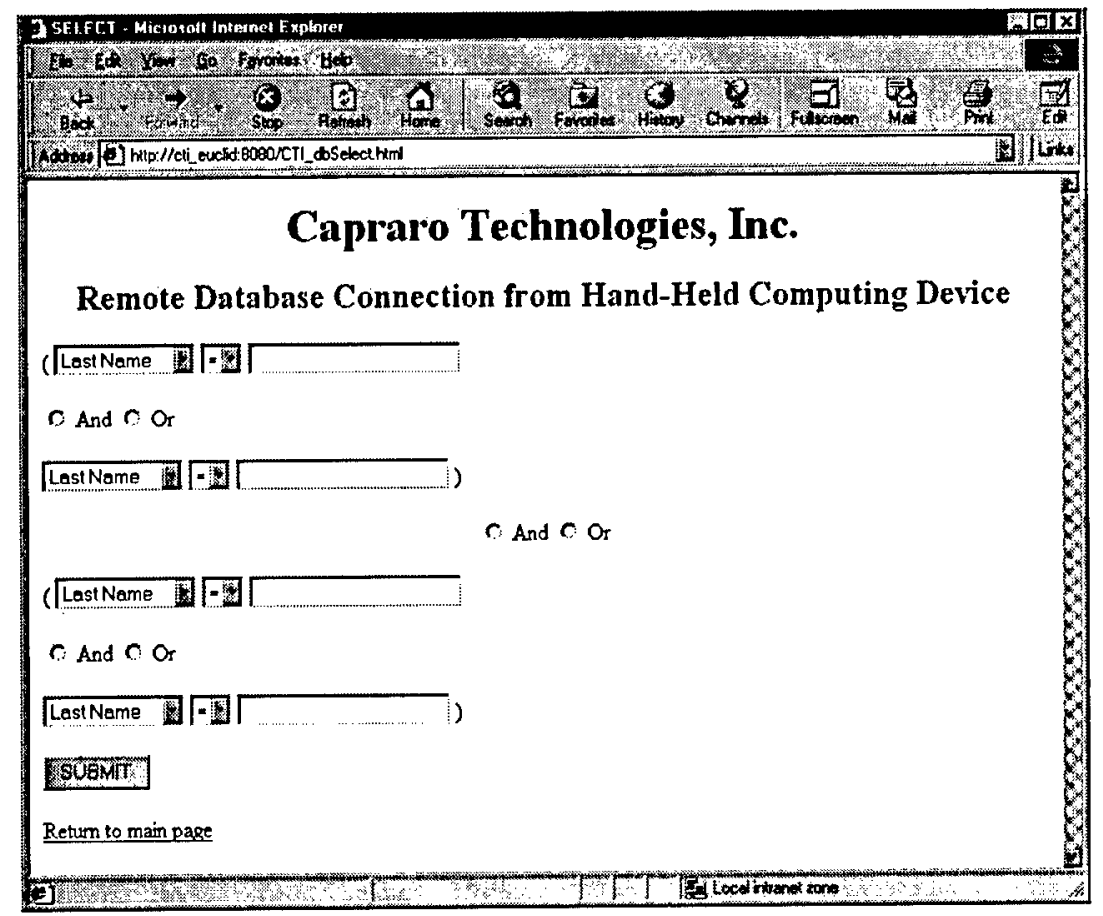

Figure 5. Database Query

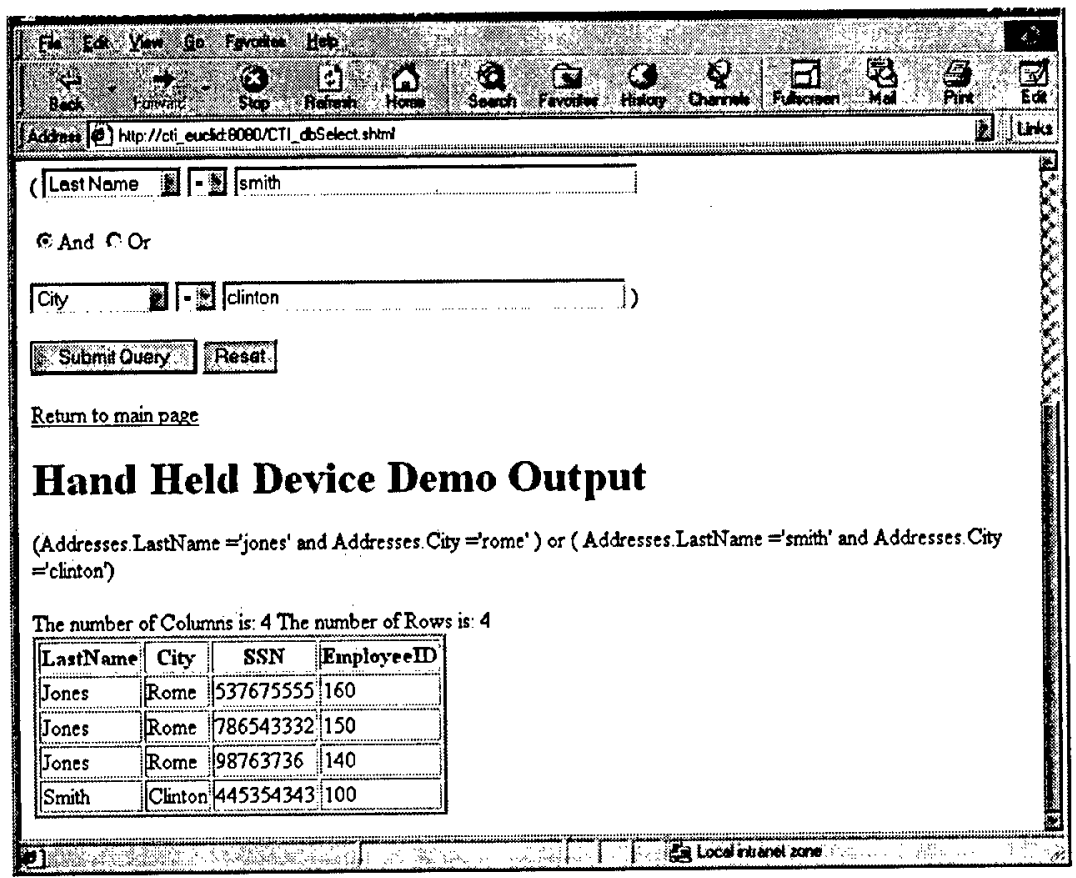

Figure 6. Database Query Result

The following screen dump, Figure 7, illustrates how a user can easily insert new data within the database. If the user makes an error, the mistake can be nulled by clicking the "Reset" button. When the input is correct the user the "Submit" button. 


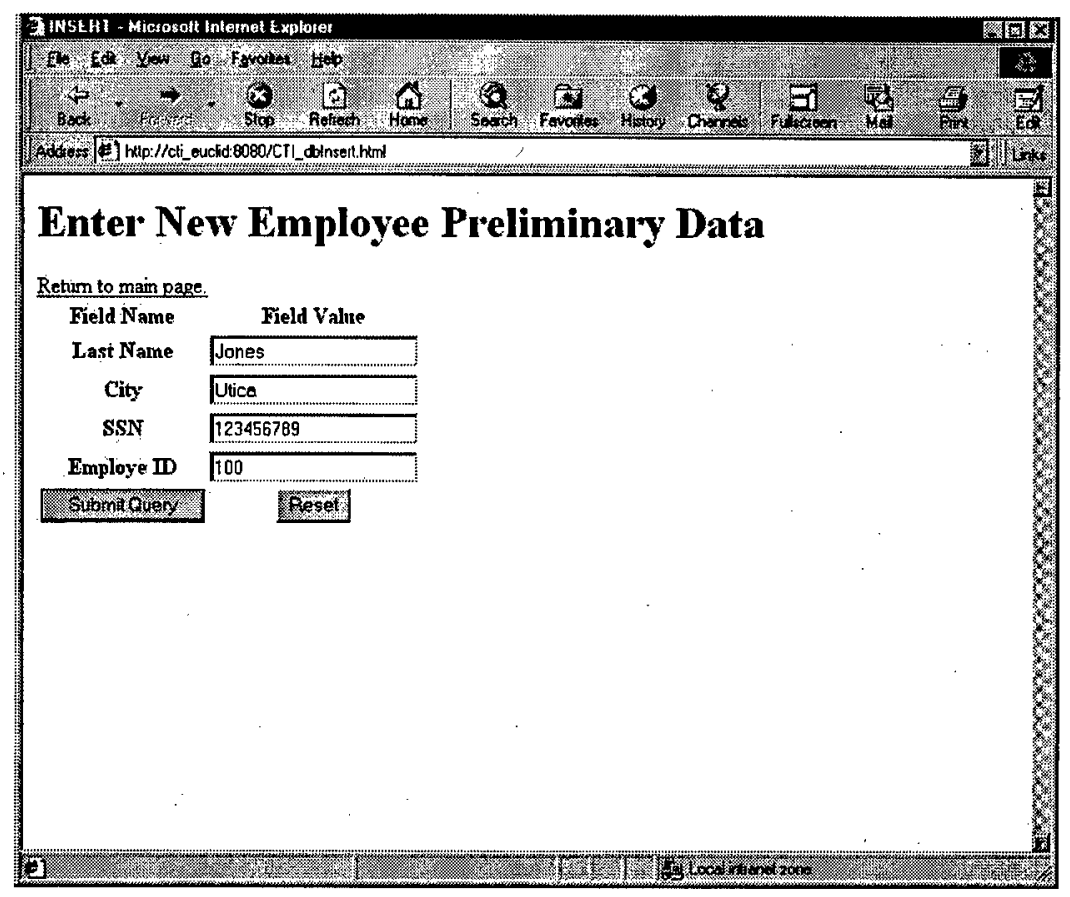

Figure 7. Database Update

These procedures are easy to understand, are conducive to "point and click", and minimize the amount of data the user has to type, or on some HCDs, write. This simplicity minimizes errors and the time required for the user to submit requests to the system.

\subsection{Preliminary Findings and New Network Technology}

The major objective for this effort was to evaluate HCDs and their ability to bring data and information to the war fighter in the field. The preliminary findings indicate that these devices have great potential as a method to bring the data and information that are available within a Government Internet to a person located almost anywhere. As these devices add more processing power and memory, some or all of the processing performed on the network can be downloaded to the devices. The Network, Browser, and Java (NBJ) paradigm is ideal for integrating these devices within the PC, workstation, and mainframe world. The demonstration above was performed using some Beta software tools. As this effort progressed these software tools have become commercially available. It is only a short time until these devices will appear throughout the world similar to the cellular phone or calculator. The mingling of phone technology, computing, e-mail, television, and eventually video collaboration is only time and bandwidth restrictive, given the rate in which processing speed, communications, and memory technology is advancing.

The NBJ paradigm will make the HCD "everyone's" machine of choice for remote computing. These devices are application independent. They can accept handwriting 
as input and/or a keyboard. Some will accept scanning devices for inputting bar codes. They can display map data and provide location coordinates with connections to a Global Positioning Satellite (GPS) system. The NBJ paradigm allows the user to retrieve the latest in software updates each time one logs into the system. This technology will allow the military to deploy troops and reduce the "footprint" of their deployment by reducing the amount of documents required in the battle field. The war fighter can always retrieve the latest information from the network rather than transporting maps, books, manuals, etc. into the field.

There are also some enhancements that are needed. Most of these devices are not "hardened" or rugged enough to withstand the varied environments the military requires. It would be beneficial if there was a standard way to use these devices to display data from different and varied sensors. Speech intelligibility would be useful in some situations. It would be beneficial in some situations if they had a larger screen with more resolution, that could be folded away when not in use. Some people would like a larger keyboard that also could be folded away.

Accessing very large databases, information sources, and knowledge bases resident on a network will require more advanced software. Integration of these data, information, and knowledge bases is not enough, intelligent based tools to help the user is required. Each user needs an intelligent personal assistant, a person's "Alter Ego," that can be tailored on the fly.

$\mathrm{HCD}$ users will require various forms of help that will reside on the network.

- The help must be all-inclusive and easy to understand.

- The processing must be transparent to the user. Intelligent software will diagnose and solve problems without interrupting the user's processing.

- Users will require the ability to utilize and manage their database. This ability includes an ad hoc query capability, and methods to add and/or delete attributes.

- Users will need to understand the results of their queries and the performance of knowledge bases. The intelligent architecture must be able to explain its answers.

- The intelligent architecture must have the ability to drill down through varied data sources, mine databases, and perform knowledge discovery.

Figure 8 shows an example architecture that illustrates the integration of multiple data sources (in this case, resident databases in DBMSs) and providing results to the war fighter. 


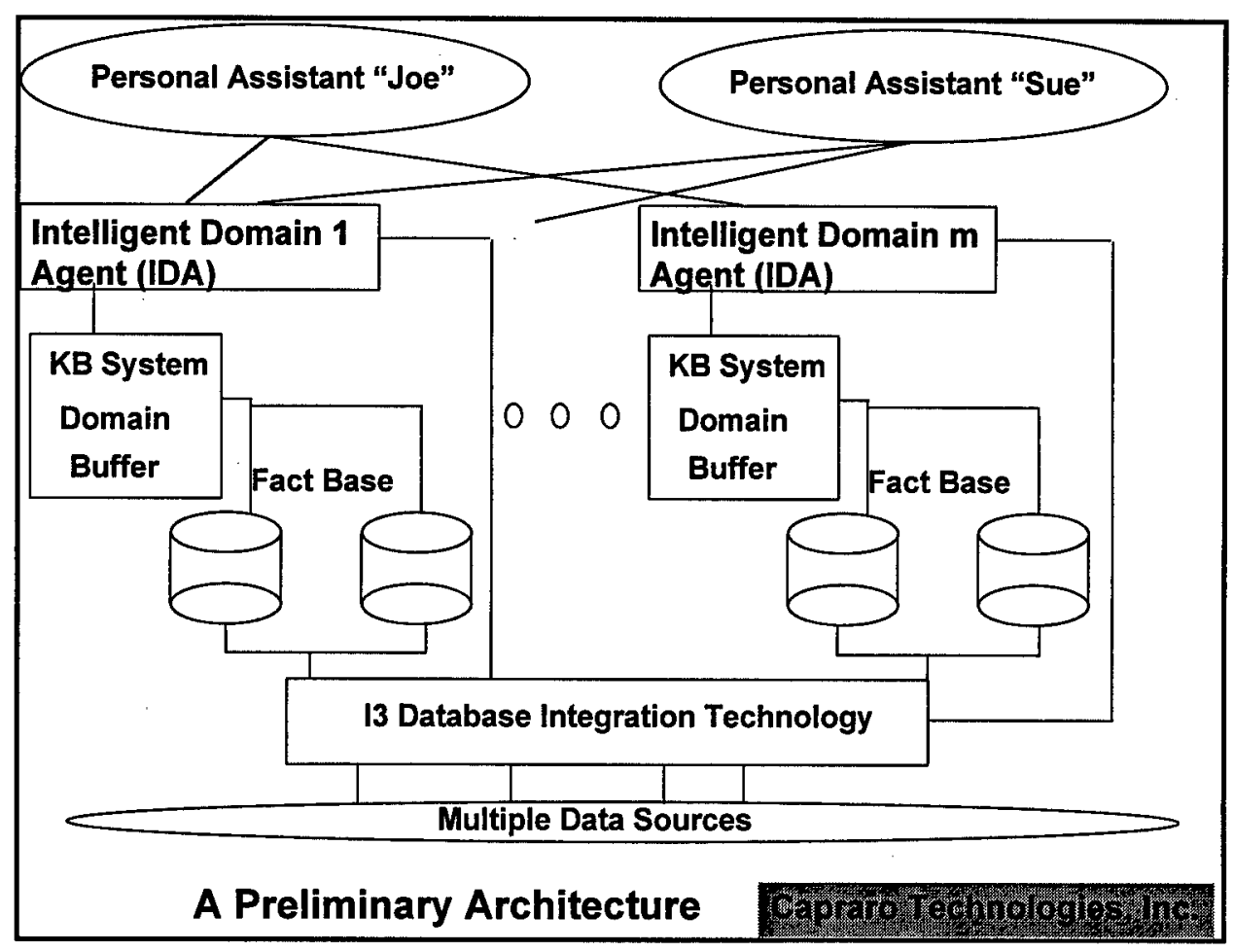

Figure 8. A Preliminary Architecture

The architecture will have intelligent software that fulfill the needs for the individual war fighter. If a request is submitted for instance of the network from a HCD then the alter ego or personal assistant will process the request differently than if the war fighter submitted the same request from a workstation with a T1 communications line. The system will assess the complexity of the request, determine its time required to perform the task, and then download the result for presentation to the user in a customized format for the computing device and available bandwidth. The system may respond to the user with an estimate of the time to respond and with a method of response, rather than just responding if the user is entering the system from a different device or bandwidth than normal. It may suggest that the user be alarmed when it is complete or have the results displayed in a graph rather than communicate the raw data. It may suggest that the raw data be sent to an e-mail address and accessed later from a more powerful computing device. The personal assistant will also optimize performance based upon the processing performed on the net, on the HCD, and the amount of bandwidth, processing speed and memory available on all the processing nodes involved. 


\section{MISSION \\ OF \\ AFRL/IFORMATION DIRECTORATE (IF)}

The advancement and application of information systems science and technology for aerospace command and control and its transition to air, space, and ground systems to meet customer needs in the areas of Global Awareness, Dynamic Planning and Execution, and Global Information Exchange is the focus of this AFRL organization. The directorate's areas of investigation include a broad spectrum of information and fusion, communication, collaborative environment and modeling and simulation, defensive information warfare, and intelligent information systems technologies. 